\title{
Developmental Biology: Preventive Medicine for Neonatology ${ }^{1}$
}

\author{
MERTON BERNFIELD ${ }^{2}$ \\ Stanford University School of Medicine, Department of Pediatrics, Stanford, California 94305-5119
}

Neonatology is a new discipline and, by all measures, it is a successful enterprise. Every day we see improvements in technology in our neonatal intensive care units. These practices, together with improved nursing and obstetric care, are in large part responsible for the decline in neonatal mortality rate that has occurred over the last 25 years.

Why then do we need preventive medicine for neonatology? Preventive medicine attempts to define etiologies and to modify the environment to reduce the incidence of disease. I will focus on some ways that developmental biology can be preventive medicine for neonatology: how an understanding of the biology of development can prevent disease in the newborn, and how training in developmental biology can prevent problems for the discipline.

\section{MAJOR CAUSE OF INFANT MORTALITY IN UNITED STATES IS PREVENTABLE}

What are the problems for the newborn? These are illustrated by examining the causes of infant mortality in this country. The major cause has been immaturity associated with low birth wt (LBW) (1-4). With improvement in neonatal intensive care, especially in the decade from 1964 to 1974 , the proportion of deaths due to immaturity has fallen, but that due to congenital anomalies has increased (Fig. 1). Most importantly, the overall rate of mortality is decreasing. Nevertheless, the United States is 19 th in infant mortality in the world (5). I cannot discuss preventive medicine for neonatology without acknowledging this most preventable cause of infant mortality.

So, before discussing developmental biology, we must ask why, despite the excellent advances in neonatal medicine, the US infant mortality is so high. The answer is that there is a very high incidence of low and especially very low birth wt (VLBW) infants in this country. LBW infants, $<2500 \mathrm{~g}$, have a 40 -fold, and VLBW infants, $<1500 \mathrm{~g}$, have a 200 -fold greater mortality rate than normal birth wt babies (6). These are premature and smallfor-gestational-age infants.

In the United States, the rate of VLBW, as well as LBW, is markedly higher in blacks, leading to a 2-fold greater infant mortality among blacks than whites (7). In 1978, a national goal was established whereby the infant mortality rate in whites and blacks would be equivalent by 1990 (8); however, we will not meet that goal. Indeed, since that time, the level of federal funding for maternal and child health has fallen (9) (Fig. 2). Therefore, although the chance of a LBW baby surviving in the United States is nearly as good as anywhere else, because we have many such infants, we have a high infant mortality rate.

I hope to convince you that understanding developmental biology can prevent problems for the newborn, but it is clear that the best preventive medicine for neonatology must include lowering the incidence of LBW, which, in this country, means

Supported in part by NIH Grant HD 06763. 1988.

' Presented at the American Pediatric Society Centennial Symposium, May 3,

${ }^{2}$ Present address: Merton Bernfield, M.D., Joint Program in Neonatology, Harvard Medical School, Boston, MA 02115. primarily lowering this rate in blacks. The cause of this ethnic disparity is unclear but there is good evidence that it may, in part, be due to lack of adequate prenatal care. Adequate prenatal care is known to be associated with increased birth weight and improved birth outcome $(10,11)$.

Recently, Jann Murray, then a Stanford undergraduate, and I postulated that if women had equal opportunities for prenatal care, adequate prenatal care would be associated with a greater reduction among blacks of the incidence of LBW and VLBW (12). We focused on the Kaiser Hospitals in California because members of this health maintenance organization have unrestricted access to prenatal care and, possibly because only the employed are members, the black and white mothers in Kaiser were essentially equivalent in several measures of socioeconomic status.

The rates of LBW and VLBW in this cohort were still higher in blacks than whites. But, the rates decreased with increasing levels of prenatal care, as measured using the definitions of the Institute of Medicine (Fig. 3). As we hypothesized, adequate care was associated with a greater reduction in very LBW among blacks than among whites. Indeed, the relative risk was reduced 3.6-fold for black mothers and 2.1-fold for white mothers. We found a similar difference in relative risk for LBW and for smallfor-gestational-age babies (12).

Therefore, even in this low risk population, early and consistent prenatal care is associated with a greater improvement in the birth wt of black babies. These data suggest that improving prenatal care in populations that are at even higher risk would result in a greater improvement in birth outcome.

How can we apply these findings to preventive medicine for neonatology? Pediatricians do not provide prenatal care; but we must be concerned about Federal policies for funding educational and health care programs for mothers and for effective delivery of prenatal care. Moreover, as citizens, we can vote to ensure that the prevention of LBW again becomes a national priority.

Now, let's look at the other causes of neonatal mortality. The major causes are prenatal but there are also perinatal and postnatal causes. To prevent these, we must understand their prenatal etiology and that means their development. Therefore, I will now describe some basic principles of development, define effector molecules that control development, and then provide examples where knowledge of developmental biology can help prevent some prenatal, perinatal, and postnatal diseases of the newborn.

\section{BRIEF DESCRIPTION OF PRINCIPLES OF DEVELOPMENTAL BIOLOGY}

Development involves the organization of cell populations into unique arrangements. The fertilized egg undergoes cleavage, becomes an implanting blastocyst, and then, in what Lewis Wolpert called the most important event in our lives, cells move during gastrulation to form mesenchyme and a three-layered embryo. Neurulation, in which the neural folds close, then lays down the basic body plan.

Much work to understand the genetic control of early devel- 




Fig. 1. Changing causes of infant mortality, 1954-1987. In the past, the major cause of infant mortality was immaturity associated with LBW. In the past three decades, especially in the decade from 1964 to 1974 , these deaths have declined and those related to congenital anomalies have increased. (From ref. 1, including provisional data for 1987.)

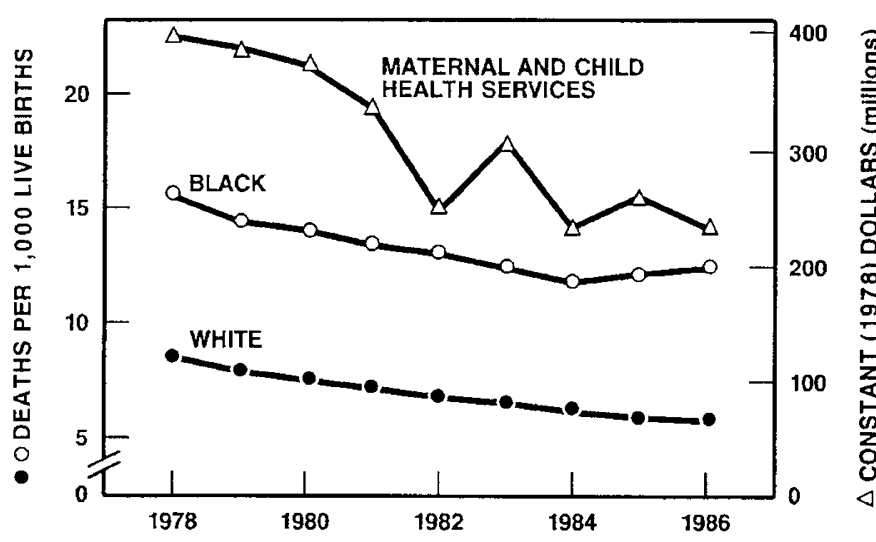

Fig. 2. Trends in neonatal mortality and in U.S. government support for maternal and child health services, 1978-1986. The level of Federal funding for maternal and child health fell during this period. At the same time, the reduction in neonatal mortality rates for black infants lessened and then increased. (From refs. 1 and 6. )
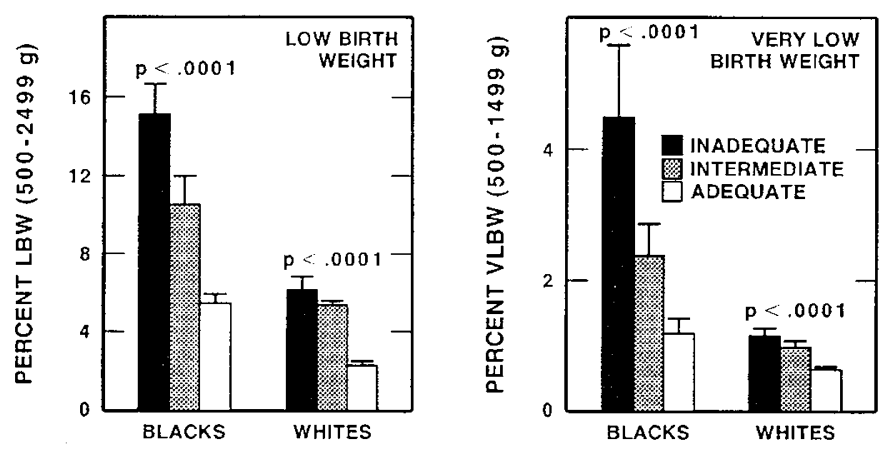

LEVEL OF PRENATAL CARE

Fig. 3. Relation between prenatal care and LBW and VLBW among blacks and whites in the California 1978 Kaiser cohort. Prenatal care was associated with improvement in birth outcome for both races but the decrease was greater for blacks than for whites as assessed by weighted analysis of covariance of births in Kaiser-Permanente Hospitals in 1978. The $p$ values are based on Mann-Whitney-Wilcoxon analyses for trends in ordered categories. Blacks represent 3928 births and whites represent 27944 births. (Modified from ref. 12.) opment has concentrated on the fruit fly, Drosophila melanogaster, and the nematode worm, Caenorhabditis elegans, because of the ease of identifying mutations in early developmental genes. These studies reveal a hierarchy of developmental genes in which mutations in one set of genes affect the expression of other genes lower in the hierarchy. For example, a single complex of genes called homeotic genes controls the development of segmentations in the fly. These homeotic genes code for a DNA-binding protein sequence that is highly conserved in the genomes of other segmented animals, including humans (13). However, it is not known how the genes control morphogenetic events or whether any of the genes regulate the expression of the effector molecules that control cell behavior during development.

Much of what we know about developmental mechanisms relevant to humans, however, comes from studies of the development of the mouse. Recently developed methods for the isolation, molecular analysis, and germ line reintroduction of mouse genes should provide new information on the genetic control of early development in mammals.

Development is a cellular process that involves only a few cellular behaviors (Fig. 4). Developing tissues contain cells in only two types of arrangements; cells in sheets and cells in clusters. The cells in sheets are the epithelia, whereas the cells in clusters are the familiar mesenchymal cells. These cells can undergo only a limited number of behaviors, they have a limited repertoire: cells can adhere to one another and to their underlying extracellular matrix; cells can change shape and when they do, they move; cells can grow and increase in number; and, finally, cells can specialize and acquire new functions during differentiation.

The general principle is that all of development, including the morphogenesis of specific tissue forms, can be understood in terms of these two cellular organizations and a few types of cellular behavior.

What causes these changes in cell behavior? All cells have the hardware, the organelles and enzymes, for these behaviors. The changes depend on how the cells are organized and how they are regulated. For example, every cell has an actin-containing cytoskeleton that acts as a cell's internal muscle to change cell shapes. When isolated cells are on a surface, actin-mediated changes in cell shape pull the cells along, causing locomotion. When cells are linked in a sheet, an actin-mediated, purse-string-like effect at one end of the cell causes the entire tissue to bud and form branches or ducts.

How are these developmental cell behaviors regulated? According to Sydney Brenner, there are two basic strategies. In one, genetic information dictating behavior is passed from parental cell to daughter cell. This is the so-called cell lineage model where all cells dance the same tune. In the other, the behavior of the

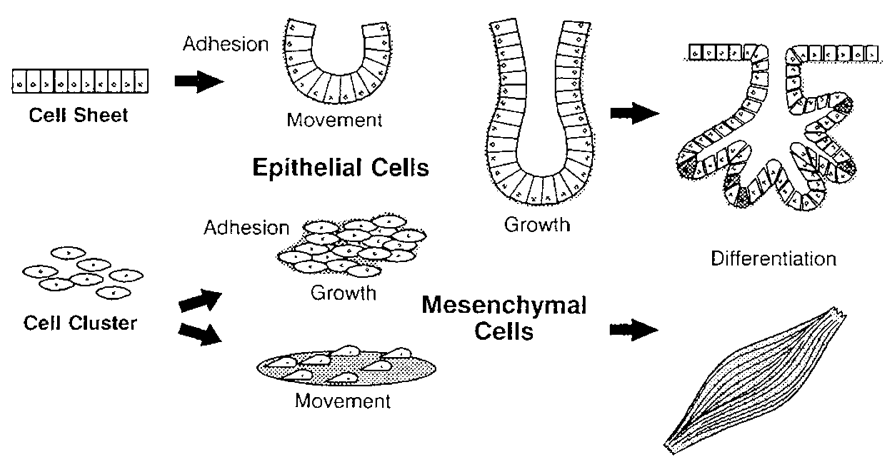

Fig. 4. Development involves a limited repertoire of cellular behaviors. Cells in developing tissues are in two types of arrangements, sheets or clusters. These cells, which represent epithelia and mesenchyme, undergo adhesion, change in shape leading to movement, cell proliferation, and differentiation. These behaviors are regulated by cell interactions that are mediated via extracellular matrix, cell adhesion molecules, and growth factors. 
daughter cells is influenced by other cells. This is the cell interaction model. (The first strategy is the so-called European scheme, where the daughter cells behave as their parents dictate. The second is the so-called American scheme. Here, the daughter cells do what their neighbors expect).

The behavior of cells during development is highly integrated and the integration involves both strategies: a cell's responses to its neighbors will depend on its lineage and a cell's prehistoryhow it was previously influenced-will determine its current behavior. Knowledge of cell lineages in mammalian development is not extensive, but there is much evidence for cell and tissue interactions. Indeed, most organs rise as a consequence of interactions between epithelial and mesenchymal cells.

\section{EFFECTOR MOLECULES OF CELL INTERACTIONS}

Recently, remarkable advances have been made in identifying the effector molecules in cell interactions. These are extracellular matrix, cell adhesion molecules, and "growth" factors that interact with receptors at the cell surface and transduce signals inside the cell. I would like to take a brief look at these molecules to discuss how they interact in developmental events.

Although the extracellular matrix is often thought to be the stuffing between cells, it provides the substratum or surface on which the cells lie (Fig. 5). Epithelial and mesenchymal cells produce distinct matrix components but they share common features: Mesenchymal cells produce interstitial matrix and the epithelial cells produce a basal lamina $(14,15)$. Each matrix contains a collagen type as the major component, types I, III, and $\mathrm{V}$ in the interstitial matrix and type IV in the basal lamina; each contains a cell-binding glycoprotein, fibronectin, and laminin. The matrix is composed of very large molecules that are extensively cross-linked, producing a composite that is insoluble and adjacent to the cells. For example, hyaluronic acid, a glycosaminoglycan (mucopolysaccharide), that is a very large molecule that binds to other proteoglycans, is hydrated extensively and occupies giant volumes.

The next class are the cell adhesion molecules, the "glue" molecules at the cell surface that stick cells together (16) (Table 1). They are of two general types: calcium dependent and calcium independent. They appear to act by binding to an identical molecule on the adjacent cell surface to form intercellular bridges at sites of cell contact. In this way, they segregate populations of

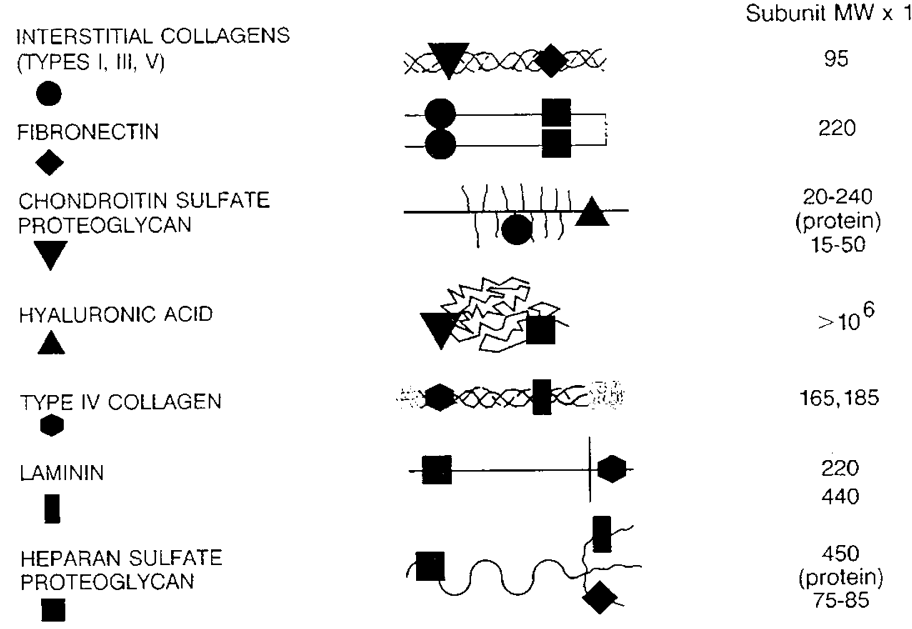

Fig. 5. Major extracellular matrix components at tissue interfaces. Major components of extracellular matrices are collagens, adhesive glycoproteins, and proteoglycans. Mesenchymal cells produce an interstitial matrix of predominantly collagens (types I, III, IV), fibronectin, chondroitin sulfate proteoglycan, and hyaluronic acid, whereas epithelial cells produce a basal lamina (basement membrane) of predominantly type IV collagen, laminin, and heparan sulfate proteoglycan. The symbols represent the binding interactions among these components. cells and assemble cell junctions. Much experimental evidence indicates that these cell adhesion molecules link cells together. The extracellular matrix, however, is distributed over many cells, allowing it to integrate cellular events into outcomes at the tissue level.

The other class of effector molecules are the growth factors, which are also called the signalling peptides (Table 2). Originally identified by their action on the proliferation of cells in culture, they are now known to have a much wider range of action. Recent work indicates, for example, that relatives of transforming growth factor- $\beta$ (TGF- $\beta$ ) and fibroblast growth factor (FGF) are involved in establishment of the dorso-ventral axis in amphibia (17). Certain of these peptides or their receptors have a close but poorly understood relationship to cellular oncogenes, implying that the oncogenes have a physiologic role during development (18). For example, a platelet derived growth factor (PDGF) chain is encoded by the sis oncogene and the epidermal growth factor (EGF) receptor is encoded, in part, by the $\operatorname{erb}-B$ oncogene.

The response to these signaling peptides appears to depend on the state of differentiation of the target cells and on the context set by the presence of other peptides. For example, TGF- $\beta$ stimulates the growth of certain fibroblasts in the presence of PDGF, but inhibits their growth if EGF is present. Sporn and Roberts (19) have suggested that combinations of peptides may act as a cellular signaling language in which integration of a variety of signals produces the context of the messages. Several of these peptides bind as active forms to extracellular matrix components, which keeps the peptide acting locally, adjacent to the cells.

To this point, I have indicated that development is a cellular process, that there are overlapping strategies, and that there are effector molecules that control cell behavior during development. Now, I want to give an example of how such molecules can interact with cells and conclude with instances where developmental biology can provide insights into diseases of the newborn.

Many organs arise by interactions between epithelium and mesenchyme. One way that the mesenchyme influences the epithelium is by remodeling the basement membrane, the matrix that lies between these tissues (20). The mesenchyme deposits interstitial matrix and also enzymatically degrades the basal lamina. Basement membrane remodeling is involved in the morphogenesis of several branching epithelial organs, such as the lung, kidney, prostate, and biliary tree, and occurs during the initiation and regulation of capillary growth.

However, how do the cells recognize and respond to the matrix changes? Although specific receptors for matrix molecules are at the cell surface, they can't act as typical cell-surface receptors because of the insolubility and large size of the matrix composite. Also, the matrix is continuously present adjacent to the cell and does not change its concentration and, therefore, must have a unique mechanism for generating a transmembrane signal.

To assess possible matrix receptors, we have used a simple morphogenesis system, placing sheets of cultured mammary epithelial cells into three-dimensional gels of Type I collagen. In these gels, the cells form duct and tubule-like structures, resembling the normal in vivo morphogenesis of the mammary gland. We added a number of soluble analogs of normal matrix materials to the cultures. When heparin was added to such cultures, it prevented morphogenesis; the cells remained as a sheet, held together by E-cadherin, a cell adhesion molecule. However, within $24 \mathrm{~h}$ after removing the heparin, the cells formed ducts again. This suggested that heparin, a well-known glycosaminoglycan, blocked the attachment of the cells to the collagen so ducts could not form, implying that a heparin-like molecule at the cell surface might be involved.

We analyzed these cells for such a molecule and found that a proteoglycan, a protein containing glycosaminoglycan chains, was responsible (21). We have named this proteoglycan syndecan, from the Greek, syndein, "to bind together." Syndecan contains two types of glycosaminoglycan chains and is an integral membrane protein (Fig. 6). Syndecan behaves as a receptor 
Table 1. Cell adhesion molecules (CAM) in mammalian development

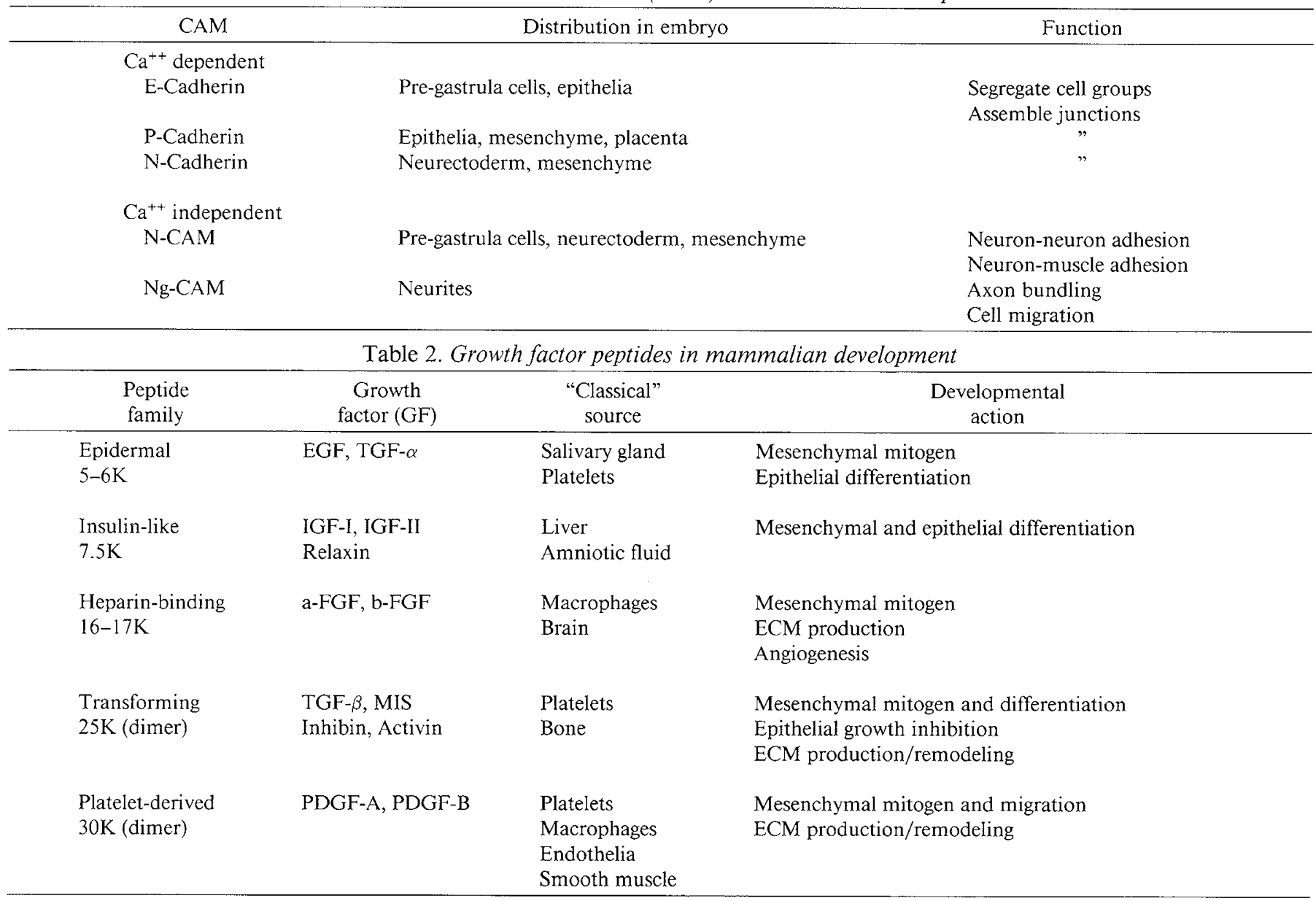

Associates with cytoskeleton when ectodomain cross-linked

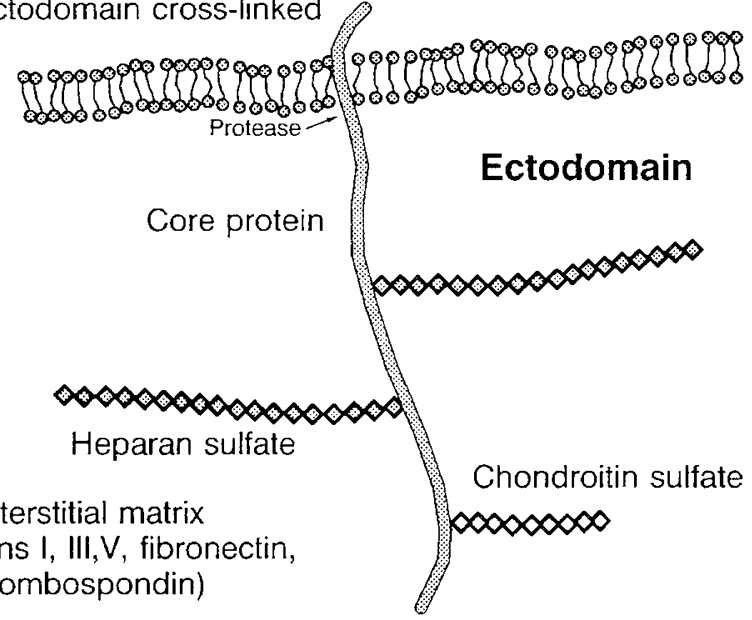

Fig. 6. Schematic structure of syndecan, a cell surface proteoglycan. Syndecan is an integral membrane proteoglycan that contains a $31 \mathrm{kD}$ core protein with a small cytoplasmic domain and an extracellular domain (ectodomain) bearing both heparan sulfate and chondroitin sulfate chains. The cytoplasmic domain associates intracellularly with the actin-containing cytoskeleton when the ectodomain is cross-linked at the cell surface. The ectodomain binds interstitial matrix components. A protease-susceptible site adjacent to the plasma membrane is cleaved when cells change shape, releasing the ectodomain and breaking a transmembrane linkage of the cytoskeleton to the matrix. (From ref. 21 and sequence data in ref. 22.) specific for the interstitial matrix produced by mesenchymal cells. It binds cells via the heparan sulfate on its extracellular domain, or ectodomain, to interstitial collagens and fibronectin, but not to basal lamina components. It associates intracellularly with the actin cytoskeleton when its ectodomain is cross-linked at the cell surface. When cultured cells are forced to change shape, they shed the ectodomain intact, releasing the same molecule that can be cleaved from the cell surface by proteases. In tissues, syndecan is polarized on the basolateral surfaces of epithelial cells, the sides of the cells that are adjacent to the matrix, exactly where it should be as a matrix receptor.

To assess the actual structure of syndecan and understand its regulation, sydecan cDNA was cloned from libraries made from the mammary epithelial cells and sequenced (22). The derived amino acid sequence is unique and shows several regions of interest: a putative transmembrane domain; the cytoplasmic domain which interacts with the actin cytoskeleton and contains the only potentially phosphorylatable tyrosines, the substrates for certain protein kinases; a single dibasic protease susceptible site, immediately adjacent to the transmembrane domain, where the ectodomain is apparently shed when cells change shape, implying the involvement of a shape-dependent surface protease; two regions in the ectodomain where glycosaminoglycan chains can be added, which could explain why syndecan has two different types of these chains; and both $5^{\prime}$ and $3^{\prime}$ regions of the cDNA outside the coding region, which have sequences substantially identical to the analogous regions of the human insulin receptor cDNA, suggesting a similar mode of regulation.

How does syndecan function during development? Clues have been obtained by inhibition studies and by examining its distribution seen with monoclonal antibodies (23). For example, on the head of an 8-d mouse embryo, syndecan is on the basolateral cell surfaces only of the surface epithelium and is weakly present 
on the cells of the underlying optic stalk, an extension of the brain which will subsequently induce the lens in the surface epithelium. On a 9.5-d embryo, the optic stalk has become round and has lost its stain. The overlying epithelium, thickened to form a placode, has also lost its strain. This and other studies indicate that syndecan is lost, sometimes transiently, when tissues change shape. Syndecan appears to stabilize the form of epithelial cell sheets by transducing a signal from the matrix to the cytoskeleton. We have called this process "matrix anchoring" (21).

Classically, receptors are involved in opening ion channels, producing second messengers and endocytosing ligands. In matrix anchoring, the cell surface receptor binds to an insoluble matrix; the matrix cross-links the receptor in the plane of the membrane, linking it to the intracellular cytoskeleton, causing a change in cell shape. The matrix-syndecan-cytoskeletal complex is immobilized at basal cell surfaces and this physical linkage to the matrix stabilizes the cell sheet.

This mechanism implies that changes in cell shape can be induced by either disruption of the matrix or loss of the matrix receptor itself. For example, in the case of lens induction, local remodeling of the basement membrane could account for the loss of syndecan and the change in epithelial shape. This is an example of how matrix molecules can regulate cell behavior during development.

\section{DEVELOPMENTAL BIOLOGY AND DISEASES OF NEWBORN}

Developmental biology can help us understand diseases of prenatal, perinatal, and postnatal origin. These are examples using animal models and cell culture models. Knowledge of the effector molecules operating during development can be used to understand and study iatrogenic problems occurring in the nursery.

First, I will discuss prenatal events and the major causes of neonatal mortality (Fig. 7). The importance of congenital anomalies to neonatology is increasing, but their etiology is often unclear. The major ones, congenital heart defects, genito-urinary anomalies and neural tube defects, are postulated to have a multifactorial etiology, meaning that they are caused by a genetic predisposition combined with an unknown environmental influence.

This postulated etiology is not readily translated into molecular mechanisms, but clues can be obtained from normal development. For example, neural tube defects result from failure of closure of the neural folds. From studies of normal embryos undergoing neurulation in whole embryo culture, Andrew Copp and I (24) showed that hyaluronic acid accumulates along the neuraxis in greatest amounts at the site and time before neural fold closure. That is, radiolabeled hyaluronate was greatest in the open posterior neuropore when compared with the closed neural tube, hind brain, and forebrain, or limb buds. This suggested

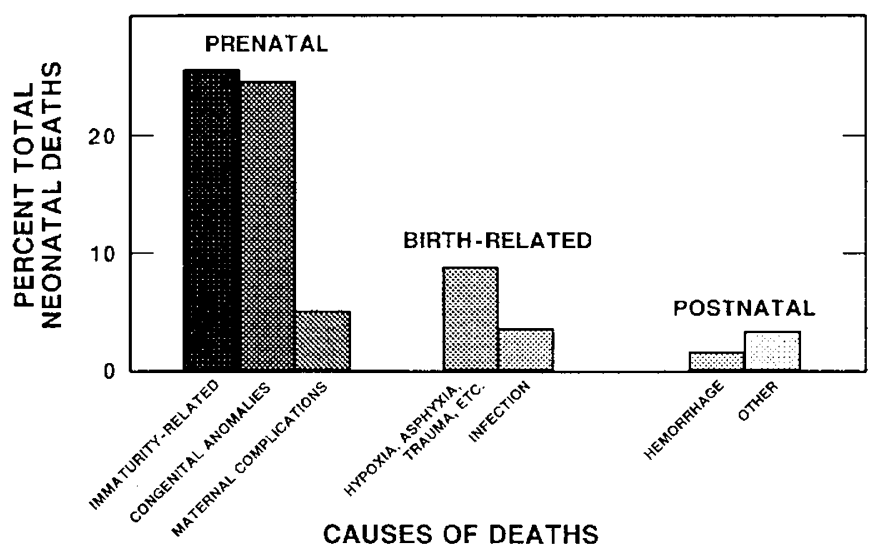

Fig. 7. Prenatal events are the major causes of neonatal mortality (1985). (Data from ref. 1.) that hyaluronate might be involved in neural fold closure, possibly accounting for the copious hyaluronate accumulation seen beneath the neural tube and surrounding the notochord.

Therefore, we turned to an animal model, the curly tail mouse, that produces neural tube defects that resemble those of humans in inheritance, prevalence of lumbosacral defects, and association with hydrocephalus. Animal models of anomalies can be helpful, but often these are studied retrospectively; that is, observations on malformations are compared with those on unaffected embryos, resulting in possible complications from the developmental anomaly itself. Needed are ways to compare future abnormal embryos before the defect occurs.

Importantly, in the curly tail mouse, future normal can be distinguished from future affected embryos before neurulation by the size of their posterior neuropore; at 27-29 somites, affected embryos have a 3- to 4-fold longer opening. Embryos at this stage can be graded as future normal or abnormal and then labeled with radioactive precursors as whole embryo cultures during which time neurulation proceeds. In future abnormal embryos, there is a $25 \%$ reduction in the amount of newly synthesized hyaluronate in the posterior neuropore region specifically. However, when these regions were examined by autoradiography, the difference between future normal and abnormal embryos localized to the basement membrane region underlying the open neuropore; the difference was abolished by treatment with a highly specific hyaluronidase. Therefore, embryos developing a spinal neural tube defect, but not their normal litter mates, accumulated reduced amounts of hyaluronate beneath the neurepithelium and around the notochord before the time that the neuropore would have normally closed (25). This abnormality, if reflected in the amnotic fluid of humans, could potentially be used to detect neural tube defects at about $4 \mathrm{wk}$ of human development.

Obviously, further work must be done to search for a molecular mechanism for the neural tube defects in these mutants. The important principle, however, is that knowledge of developmental mechanisms may allow prospective study of other animal models for congenital anomalies.

Now, let's turn to an example of a perinatal problem that can be shown in cell culture. A significant problem in neonatology, because of its greater than $50 \%$ mortality, is the syndrome of persistent pulmonary hypertension. Seen most frequently in term or postterm infants with severe meconium aspiration or perinatal asphyxia, it is difficult to treat successfully because of the excessive extension of smooth muscle into peripheral pulmonary arteries, originally noted by Lynne Reid and her collaborators (26). These workers showed that the pre-acinar and intraacinar arteries are not normally muscularized at birth, but that these infants and hypoxic animals have precocious muscularization of these vessels.

First with John Coulson and then with William Benitz, we pursued the hypothesis that hypoxia alters smooth muscle cell growth in cell culture (27). When smooth muscle cells from the aorta and pulmonary arteries of fetal calves were cultured, their growth was inhibited, not stimulated, by hypoxia. Moreover, they responded identically to several other growth regulators. Therefore, whatever causes the abnormal smooth muscle growth may be made and acting locally within the lungs.

Because several workers had shown that adult aortic endothelial cells produce both growth promoters and growth inhibitors, we examined materials produced by cultured fetal pulmonary artery endothelial cells. We found a growth inhibitor in conditioned medium that, upon purification, was a specific basement membrane heparan sulfate proteoglycan (28). When we checked how hypoxia might affect this process, we found that production of this proteoglycan inhibitor by the fetal pulmonary artery endothelial cells was markedly inhibited by low oxygen tensions. Much more work is needed to evaluate how the inhibitor functions, how it is regulated, and whether its production is modified by various types of endothelial injury. 
These studies suggest a tissue interaction model for the abnormality in smooth muscle cell growth seen in persistent pulmonary hypertension of the newborn. A potent inhibitor of smooth muscle cell growth is produced by the endothelial cells and presumably deposited in the basement membrane, a site strategically close to the target cells. Chronic fetal hypoxia could result in reduced production of this inhibitor, potentially allowing the growth and precocious extension of smooth muscle into the distal arterioles, leading to pulmonary hypertension.

These studies suggest that when an abnormality in one of the specific morphogenetic behaviors is suspected, it may be possible to avoid the complexities of tissue morphology and do "developmental biology in cell culture."

Finally, I would like to discuss some postnatal problems. The finely orchestrated process of tissue repair attempts to reestablish normal morphology using the same molecules that control cell behavior during development (29) (Fig. 8). Injuries to tissues can result in disruption of the epithelial layer, its basal lamina, the underlying stroma, and capillaries. Blood extravasates with degranulation of platelets, releasing TGF- $\alpha$, TGF- $\beta$, and PDGF, the keys to wound repair. Various chemoattractants produced during the ensuing coagulation cause the invasion of neutrophils. Subsequently, the entry and activation of macrophages produces more signaling peptides. The epithelial cells, which had been firmly attached to each other and to the basement membrane, lose their cell-cell adhesion and migrate into the wound on top of fibronectin deposited on the fibrin clot.

Within days, repair occurs. Under the influence of the growth factor peptides, fibroblasts are aroused to migrate into the wound, proliferating and depositing collagens, fibronectin, and hyaluronic acid, and endothelial cells migrate and proliferate, forming new capillaries. This granulation tissue is now covered by epithelial cells that deposit a new basal lamina. Further deposition and remodeling of the extracellular matrix results in fibrosis and a scar.

Much clinical data suggest that tissue repair in the fetus and newborn differs from that in the more mature infant or adult. The most obvious differences are fewer neutrophils and most importantly, a very marked reduction in fibrosis (30). The basis for the difference in fibrosis is unclear, but could be due to endogenous signalling peptides in the tissues undergoing morphogenesis.

The reduced fibrosis in injuries repaired by the fetus and newborn contrasts with the extensive fibrosis seen in two major problems in the neonate, bronchopulmonary dysplasia and retinopathy of prematurity. These injuries lead to attempts at repair that involve excessive fibrosis, but the mechanism is wholly
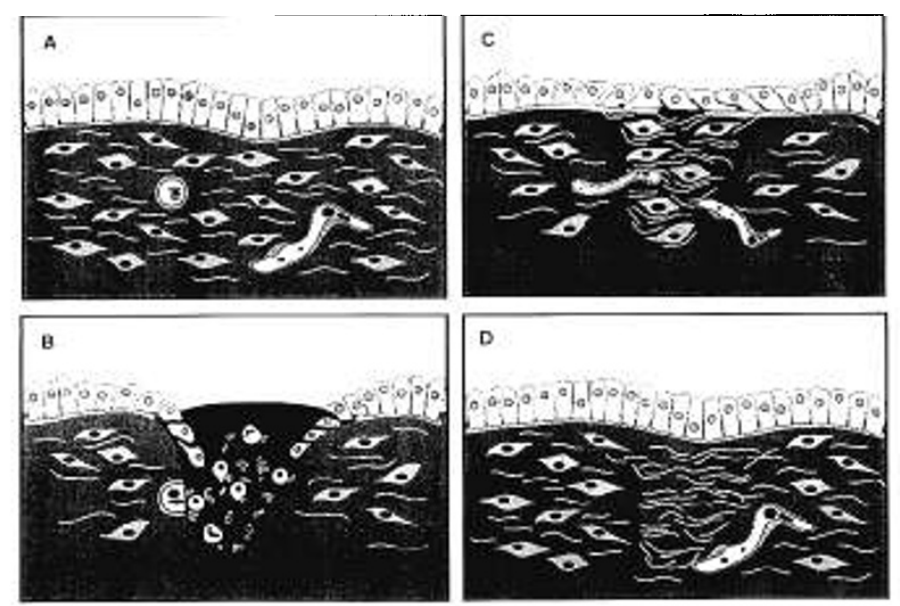

Fig. 8. Repair of injury. The sequence of events leading to reconstitution of histoarchitecture after injury involves the same molecules, viz. extracellular matrix components, cell adhesion molecules, and growth factors, that are involved in cell interactions during normal development. See text for details. unclear. Because the molecules involved in tissue repair are identical to those involved in controlling developmental cell behavior, this is another area where developmental biology could be preventive medicine for neonatology.

\section{PREVENTIVE MEDICINE FOR NEONATOLOGY}

We have discussed some possible preventive approaches for problems of the newborn. Finally, we must discuss preventive medicine for the discipline of neonatology. The success of neonatology is based, in large part, on technology and aggressive approaches to care. These have a high economic cost and so far our society has justified paying for it (31). What's in the future? Better high-frequency ventilation? More extensive use of extracorporeal membrane oxygenation? I hope not. Preventive medicine for this discipline is in educating young pediatricians to be as familiar with the cell and molecular biology of development as with pulmonary physiology, and to be able to apply this knowledge to research that is not tied to a specific method or procedure.

There is an important lesson from work on pulmonary surfactant (32). After Avery and Mead's finding of surfactant deficiency in the respiratory distress syndrome (33), chemical studies showed surfactant to contain phospholipids and proteins. Cell biologic work showed that surfactant was made in the type II pneumocyte, and developmental studies of its synthesis led Gluck to devise a prenatal test for lung maturity. Subsequent molecular biology in several labs, including those of Ballard, Flores, Jobe, Shapiro, and Whitsett, identified and isolated cDNA for surfactant apoproteins, making possible study of their genetic expression and the generation of human surfactant for therapy. The lesson is that these neonatologists knew the language of basic biology and drew from it, both conceptually and methodologically, to solve a problem. There are other major problems in neonatology and our young people must be educated to solve them. We must start early, provide role models, and build a supportive environment. It can be done.

In summary, I've tried this morning to show that the future of the care of newborns depends on developmental biology, that the future of the discipline will require individuals trained in developmental biology, and that we all must keep policy makers in this country aware of preventing LBW.

Will developmental biology be preventive medicine for neonatology? Any expert in preventive medicine will tell you that the most difficult aspect of prevention is measuring outcome.

Acknowledgments. The author thanks Audrey Bernfield, Carl Morris, and Daniel Vandersteen for their help in preparing this lecture.

\section{REFERENCES}

1. U.S. Department of Health, Education, and Welfare, Public Health Service 1954 Monthly Vital Statistics Report, Annual Summary for 1954, part 2, Vol 3, No 13, p 10, Table G

2. U.S. Department of Health, Education, and Welfare, Public Health Service 1966 Final Mortality Statistics for 1964, Vol 14, No 10, Suppl, p 11, Table 10

3. U.S. Department of Health, Education, and Welfare, Public Health Service 1976 Final Mortality Statistics for 1974, Vol 24, No 11, Suppl, p 19, Table 10

4. U.S. Department of Health, Education, and Welfare, Public Health Service 1988 Births, Marriages, Divorces, and Deaths for 1987, Vol 36, No 12, March 21, 1988, p 10, Table 8

5. Children's Defense Fund 1988 The Health of America's Children: Material and Child Health Data Book, p 5

6. McCormick MC 1985 The contribution of low birth weight to infant mortality and childhood morbidity. N Engl J Med 312:92-90

7. Institute of Medicine, National Academy of Sciences 1985 Preventing Low Birth Weight. National Academy Press, Washington, DC

8. U.S. Department of Health, Education, and Welfare, Public Health Service, Office of the Assistant Secretary for Health and Surgeon General 1979 Healthy People: The Surgeon General's Report on Health Promotion and Disease Prevention, DHEW (PHS) Pub. No. 79-55701. U.S. Government Printing Office, Washington DC, $\mathrm{p} 21$

9. U.S. Congress, Office of Technology Assessment 1988 Health Children: In- 
vesting in the Future, OTA-H-345. U.S. Government Printing Office, Washington, DC, p 44, Tables 2-8

10. Gortmaker SL 1979 The effects of prenatal care upon the health of the newborn. Am J Public Health 69:653-660

11. Quick JD, Greenlick MR, Roghmann KJ 1981 Prenatal care and pregnancy outcome in an HMO and general population: a multivariate cohort analysis. Am J Public Health 71:381-390

12. Murray JL, Bernfield M 1988 The differential impact of prenatal care on the incidence of low birth weight among blacks and whites in a pre-paid health care plan. N Engl J Med 319:1385-1391

13. Kongsuwan K, Webb E, Housiaux P, Adams JM 1988 Expression of multiple "homeobox" genes within diverse mammalian haemopoietic lineages. EMBO J 7:2131-2138

14. Martin GR, Timpl R 1987 Laminin and other basement membrane components. Annu Rev Cell Biol 3:57-85

15. McDonald JA 1988 Extracellular matrix assembly. Annu Rev Cell Biol 4:183208

16. Takeichi M 1988 The cadherins: cell adhesion molecules controlling animal morphogenesis. Development 102:639-655

17. Woodland H, Jones L 1988 Growth factors in amphibian cell differentiation. Nature 332:113-115

18. Adamson ED 1987 Oncogenes in development. Development 99:449-471

19. Sporn MB, Roberts AB 1988 Peptide growth factors are multifunctional. Nature 332:217-219

20. Bernfield M, Banerjee SD, Koda JE, Rapraeger AC 1984 Remodeling of the basement membrane as a mechanism of morphogenetic tissue interaction. In: Trelstad R (ed) The Role of Extracellular Matrix in Development, The 42nd Symposium of the Society for Developmental Biology. Alan R Liss, New York, pp 545-572

21. Rapraeger A, Jalkanen M, Bernfield M 1987 Integral membrane proteoglycans as matrix receptors: role in cytoskeleton and matrix assembly at the epithelial cell surface. In: Wight TN, Mecham RP (eds) Biology of Extracellular Matrix, Vol II, Biology of Proteoglycans. Academic Press, New York, pp 129-154
22. Saunders S, Jalkanen M, O'Farrell S, Bernfield M 1989 Molecular cloning of syndecan, an integral membrane proteoglycan. J Cell Biol 108:1547-1556

23. Thesleff I, Jalkanen M, Vainio S, Bernfield M 1988 Cell surface proteoglycan expression correlates with epithelial-mesenchymal interaction during tooth morphogenesis. Dev Biol 129:565-572

24. Copp AJ, Bernfield M 1988 Glycosaminoglycans vary in accumulation along the neuraxis during spinal neurulation in the mouse embryo. Dev Biol 130:573-582

25. Copp AJ, Bernfield M 1988 Accumulation of basement membrane-associated hyaluronate is reduced in the posterior neuropore region of mutant (curly tail) mouse embryos developing spinal tube defects. Dev Biol 130:583-590

26. Reid LM Davies P 1989 Control of cell proliferation in pulmonary hypertension. In: Weir EK, Reeves JT (eds) Pulmonary Vascular Physiology and Pathophysiology. Marcel Dekker, New York, pp 541-612

27. Benitz WE, Coulson JD, Lessler DS, Bernfield M 1986 Hypoxia inhibits proliferation of fetal pulmonary arterial smooth muscle cells in vitro. Pediatr Res 20:966-972

28. Benitz WE, Kelley RT, Anderson CM, Lorant DE, Bernfield M 1990 Endothelial heparan sulfate proteoglycan. I. Inhibitory effects on smooth muscle cell proliferation. Am J Respir Cell Mol Biol 2:13-24

29. Clark RAF 1985 Cutaneous tissue repair. Basic biological considerations. I. J Am Acad Dermatol 13:701-725

30. Krummel TM, Nelson JM, Diegelmann RF, Lindblad WJ, Salzberg AM, Greenfield LJ, Cohen IK 1987 Fetal response to injury in the rabbit. J Pediatr Surg 22:640-644

31. U.S. Congress, Office of Technology Assessment 1987 Neonatal Intensive Care for Low Birthweight Infants: Cost and Effectiveness (Health Technology Case Study 38), OTA-HCS-38. U.S. Congress Office of Technology, Washington, DC, p 57

32. Comroe Jr, JH 1977 Retrospectroscope: Insight into Medical Discovery. Von Gehr Press, Menlo Park, CA, pp 161-168

33. Avery ME, Mead J 1959 Surface properties in relation to atelectasis and hyaline membrane disease. Am J Dis Child 97:517-523 